\title{
A Prospective Comparison of a Noninvasive Cardiac Output Monitor Versus Esophageal Doppler Monitor for Goal-Directed Fluid Therapy in Colorectal Surgery Patients
}

\author{
Nathan H. Waldron, MD,* Timothy E. Miller, MB ChB, FRCA,* Julie K. Thacker, MD, $\dagger$ \\ Amy K. Manchester, MD,* William D. White, MPH,* John Nardiello, MD,* Magdi A. Elgasim, MBBS,* \\ Richard E. Moon, MD, CM, MSc, FRCP (C), FACP, FCCP** and Tong J. Gan, MD, MHS, FRCA, Li Ac*
}

\begin{abstract}
BACKGROUND: Goal-directed fluid therapy (GDFT) is associated with improved outcomes after surgery. The esophageal Doppler monitor (EDM) is widely used, but has several limitations. The NICOM, a completely noninvasive cardiac output monitor (Cheetah Medical), may be appropriate for guiding GDFT. No prospective studies have compared the NICOM and the EDM. We hypothesized that the NICOM is not significantly different from the EDM for monitoring during GDFT. METHODS: One hundred adult patients undergoing elective colorectal surgery participated in this study. Patients in phase I $(n=50)$ had intraoperative GDFT guided by the EDM while the NICOM was connected, and patients in phase II $(n=50)$ had intraoperative GDFT guided by the NICOM while the EDM was connected. Each patient's stroke volume was optimized using 250-mL colloid boluses. Agreement between the monitors was assessed, and patient outcomes (postoperative pain, nausea, and return of bowel function), complications (renal, pulmonary, infectious, and wound complications), and length of hospital stay (LOS) were compared.

RESULTS: Using a $10 \%$ increase in stroke volume after fluid challenge, agreement between monitors was $60 \%$ at 5 minutes, $61 \%$ at 10 minutes, and $66 \%$ at 15 minutes, with no significant systematic disagreement (McNemar $P>0.05$ ) at any time point. The EDM had significantly more missing data than the NICOM. No clinically significant differences were found in total LOS or other outcomes. The mean LOS was $6.56 \pm 4.32$ days in phase I and $6.07 \pm 2.85$ days in phase II, and $95 \%$ confidence limits for the difference were -0.96 to +1.95 days $(P=0.5016)$. CONCLUSIONS: The NICOM performs similarly to the EDM in guiding GDFT, with no clinically significant differences in outcomes, and offers increased ease of use as well as fewer missing data points. The NICOM may be a viable alternative monitor to guide GDFT. (Anesth Analg 2014;118:966-75)
\end{abstract}

$\mathrm{P}$ erioperative fluid management has long been a hotly contested topic and one that has direct bearing on patient outcomes. ${ }^{1}$ In goal-directed fluid therapy (GDFT), intravascular volume status is optimized by assessing the hemodynamic response to fluid boluses. ${ }^{2-4}$ GDFT has been associated with improved outcomes

From the Departments of *Anesthesiology and †Surgery, Duke University, Durham, North Carolina.

John Nardiello, MD, is currently affiliated with American Anesthesiology, Duke Raleigh Hospital, Raleigh, North Carolina.

Accepted for publication January 22, 2014.

Funding: Funding for this study was provided in part by Cheetah, Inc., manufacturers of the NICOM device. The first author was supported by a Duke CTSA scholarship program funded by grant number TI1 RR 024126 by the National Institutes of Health as well as the Foundation for Anesthesia Education and Research (FAER) Medical Student Anesthesia Research Fellowship. Conflict of Interest: See Disclosures at the end of the article.

This report was previously presented, in part, at the 2011 National Predoctoral Meeting on May 12, 2012, in St. Louis, MO, at the 2011 ASA meeting on October 16 and 17 in Chicago, IL, as well as at the 2012 CAS meeting on June 15 to 17 in Quebec City, Quebec, Canada.

Reprints will not be available from the authors.

Address correspondence to Tong J Gan, MD, MHS, FRCA, Li Ac, Department of Anesthesiology, Duke University, Box 3094, Suite 4315DS, Durham, NC 27710. Address e-mail to tong.gan@dm.duke.edu.

Copyright (C) 2014 International Anesthesia Research Society

DOI: 10.1213 /ANE.0000000000000182 after moderate to major surgery, with shorter hospital stays, fewer intensive care unit admissions, earlier return of bowel function, and less postoperative nausea and vomiting. ${ }^{5-10}$

Although the benefits of GDFT are well documented, the best method for assessing tissue oxygenation and intravascular volume is still intensely debated. Routine intraoperative monitoring using heart rate $(\mathrm{HR})$, arterial blood pressure (BP), and urine output (UOP) does not provide a precise measure of intravascular fluid volume or tissue perfusion. ${ }^{2}$ A pulmonary artery catheter can provide a measure of cardiac output (CO) and mixed venous oxygen saturation, as well as left and right heart filling pressures, but it is invasive and is associated with complications. ${ }^{11}$ As such, investigators have sought minimally invasive and noninvasive methods for assessing $\mathrm{CO}$.

The esophageal Doppler monitor $\left(\mathrm{EDM}^{\mathrm{TM}}\right.$, Deltex Medical, Inc., Irving, TX) has been widely used as a less invasive method for assessing $\mathrm{CO}$ and intravascular volume status. When used to guide GDFT, the EDM is associated with improved patient outcomes ${ }^{6}$; however, it has several limitations. ${ }^{12,13}$ It is semiinvasive (requires placement of a tube in the esophagus), may not be well tolerated by awake patients, requires some training to acquire expertise in using the device, and may not provide an optimal signal in some patients. 
The noninvasive CO monitor (NICOM, Cheetah Medical, Vancouver, WA) is a bioreactance-based monitor and is a sensitive and specific method for assessing fluid responsiveness. ${ }^{14,15}$ The NICOM is totally noninvasive, well tolerated in awake patients, and potentially applicable in a wide variety of patient populations. Four surface electrodes are placed across the chest, and using bioreactance technology, the device tracks hemodynamic variables including stroke volume (SV), SV variation, and CO.

No prospective studies have compared the NICOM and the EDM for guiding GDFT. As such, we conducted a prospective trial to compare GDFT guided by the NICOM versus the EDM in patients undergoing elective colorectal surgery with an Enhanced Recovery after Surgery (ERAS) protocol. Our primary hypothesis is that the NICOM does not differ significantly from the EDM in assessing baseline SV and change in SV after colloid boluses. Our secondary hypothesis is that length of stay (LOS) and postoperative complications are similar in patients receiving GDFT guided by the NICOM compared with the EDM.

\section{METHODS}

\section{Patient Population}

After approval by our IRB, written informed consent was obtained from 100 adult patients, with ASA physical status I, II, or III who were scheduled to undergo elective colorectal surgery. All patients received care according to an Enhanced Recovery After Surgery (ERAS) protocol. Briefly, the ERAS protocol includes ingestion of a carbohydrate-rich drink before surgery, placement of a thoracic epidural for intra- and postoperative pain management, minimizing intra- and postoperative opioid use, early removal of nasogastric and urinary catheters, early mobilization, and early advancement of diet. ${ }^{16}$ This protocol is designed to improve recovery after colorectal surgery and has been shown in other studies to significantly shorten overall hospital LOS. ${ }^{17,18}$ The Duke ERAS protocol is outlined in Appendix 1.

Exclusion criteria for this study included the following: age $<18$ years, emergency surgery, preoperative smallor large-bowel obstruction, significant coagulopathy, significant renal or hepatic dysfunction (creatinine or liver enzymes more than twice the upper limit of normal), congestive heart failure, esophageal pathology (to avoid complications from the esophageal probe), gastric surgery, or participation in another investigational drug study.

\section{Monitoring and Anesthetic Technique}

On arrival in the preoperative holding area, a thoracic epidural was placed (T8-9 to T11-12 interspace) using a small amount of fentanyl and/or midazolam titrated to effect. The NICOM electrodes were placed on the patient's thorax, and the monitor was calibrated. Premedication with midazolam ( $\leq 2 \mathrm{mg}$ IV) and fentanyl ( $\leq 100 \mathrm{mcg}$ IV) was administered at the provider's discretion. All patients received American Society of Anesthesiologists-recommended standard monitoring including pulse oximetry, continuous electrocardiography, noninvasive $\mathrm{BP}$, end-tidal $\mathrm{CO}_{2}$ tension, and a continuous temperature probe. When clinically indicated, a central venous catheter or arterial catheter was placed.
Anesthesia was induced with propofol (1.5-2.5 $\mathrm{mg} / \mathrm{kg}$ ) and maintained with a balanced inhaled technique. A Bispectral Index of 40 to 60 was targeted for all patients. After induction, an orogastric tube was inserted, and stomach contents were suctioned. Ventilation was instituted with 6 to $8 \mathrm{~mL} / \mathrm{kg}$ tidal volume and was adjusted to maintain an end-tidal $\mathrm{CO}_{2}$ partial pressure of 35 to $45 \mathrm{~mm}$ $\mathrm{Hg}$. Active warming was performed using forced-air warming blankets and warmed IV fluids. Intraoperative analgesia was provided using an epidural dose of hydromorphone at induction (0.4-0.8 mg), followed by an infusion of bupivacaine $(2.5 \mathrm{mg} / \mathrm{mL}, 3-6 \mathrm{~mL} / \mathrm{h})$. Neuromuscular blockade was provided by vecuronium or rocuronium and was monitored to maintain a single twitch of the train-of- 4 . In addition, patients received a prophylactic antiemetic (ondansetron, $4 \mathrm{mg}$ IV) at the end of surgery. The orogastric tube was removed before extubation. Patients' tracheas were extubated, either in the operating room or postoperatively, when they fulfilled standard clinical criteria.

\section{Fluid Management}

After the placement of an IV cannula, a lactated Ringer's solution (LR) infusion (1 L) was commenced in the preoperative holding area. After induction of anesthesia and completion of the 1-L LR infusion, a crystalloid (LR) infusion was started and maintained throughout the procedure using a dedicated infusion pump (set at $3 \mathrm{~mL} / \mathrm{kg} / \mathrm{h}$ for laparoscopic colectomy and $5 \mathrm{~mL} / \mathrm{kg} / \mathrm{h}$ for open colectomy, based on lean body weight). An esophageal Doppler probe was greased with lubricating gel and inserted orally into the midesophagus in all patients after induction of anesthesia.

Additional fluid was administered based on the algorithm illustrated in Appendix 2, which has been validated in previous studies. ${ }^{6}$ Briefly, after incision (in open cases) or pneumoperitoneum (in laparoscopic cases), patients received a $250-\mathrm{mL}$ bolus of colloid solution (Voluven, 6\% hydroxyethyl starch $130 / 0.4$ in $0.9 \%$ sodium chloride) injected in $50-\mathrm{mL}$ aliquots. If the SV increased $>10 \%$ over a 15-minute period, the patient was considered "fluid responsive" and received another fluid bolus. If the SV increased $<10 \%$ after the fluid bolus, the patient was considered "not responsive to fluids" and received another fluid bolus only when the SV decreased $>10 \%$ from the most recent baseline (Appendix 2). Hemodynamic data were recorded by research personnel before and after each colloid bolus.

This study was conducted in 2 consecutive phases. In phase I (50 patients), investigators used the EDM for guiding GDFT while the NICOM was also connected and recording hemodynamic variables. All decisions to administer fluid during phase I were based on measurements from the EDM, with no consideration given to the NICOM measurements. In phase II (50 patients), GDFT was guided by the NICOM measurements while hemodynamic variables were simultaneously recorded by the EDM. All decisions to administer fluid during phase II were based on measurements from the NICOM, with no consideration given to the EDM measurements. Phase I was conducted over an 8-month period, whereas Phase II was conducted over a 6-month period immediately after phase I. 
During anesthesia, hemodynamic variables and UOP were recorded continuously. Vasoactive medications (phenylephrine and ephedrine) were used in cases of hypotension (mean arterial $\mathrm{BP}<80 \%$ baseline), and atropine (0.3-0.6 $\mathrm{mg}$ ) was used in cases of bradycardia (HR $<45$ beats $/ \mathrm{min}$ ). At the end of the operation, the types and volumes of all fluids administered (including crystalloids, colloids, and blood products), an estimate of total UOP, and intraoperative blood loss were recorded.

\section{Postoperative Recovery}

Postoperative analgesia was accomplished using a balanced epidural local anesthetic/opioid solution (bupivacaine $[0.125 \%]$ and hydromorphone $[10 \mathrm{mcg} / \mathrm{mL}]$ ) for up to 72 hours. After patients commenced oral fluids, the epidural catheter was discontinued, and pain was managed using oral or IV opioids and opioid adjuncts. Nausea and/or vomiting were treated with ondansetron $(4 \mathrm{mg})$ or promethazine $(6.25 \mathrm{mg})$ if ondansetron had been previously administered within 6 hours of the nausea/vomiting event. All preoperative medications were recommenced when the patients tolerated oral intake.

\section{Data Collection}

After calibrating the NICOM in the preoperative holding area, a baseline $\mathrm{CO}$ and $\mathrm{SV}$ were recorded. In the operating room, $\mathrm{HR}, \mathrm{BP}, \mathrm{CO}$, and $\mathrm{SV}$ were recorded at baseline before a fluid challenge, every 5 minutes for 15 minutes after a fluid challenge, and every 15 to 30 minutes between fluid challenges. In the postanesthesia care unit (PACU), pain (at rest and with activity) and nausea were assessed hourly for up to 4 hours or until PACU discharge. In addition, the doses of all medications and volumes of all fluids received in the PACU were recorded.

Patients were visited daily in the postoperative period by independent research personnel until hospital discharge or death. During the visit, the patients were asked specific questions about adverse events, nausea, pain levels at rest and with activity, and bowel function. In addition, the types and dosages of all analgesic, antiemetic, vasoactive, or antibiotic medications were recorded. The daily volume of IV fluids (crystalloid and colloid) and UOP as well as the serum creatinine were also recorded. Postoperative care and discharge criteria are predefined using the hospital care map and protocol. The postoperative LOS was recorded, as well as any emergency department visits or hospital readmission for surgical complications within 30 days.

\section{Statistical Analysis}

Statistical analysis was performed using the SAS System ${ }^{\circledR}$ Enterprise Guide software (version 4.3, SAS, Cary, NC), and statistical significance was determined at $\alpha=0.05$. Sample size was based on a mean LOS of 5.0 days, $\mathrm{SD}=3.0$. It was estimated that 50 patients in each group would provide $80 \%$ power to demonstrate the hypothesis of equivalence, that the mean LOS in the 2 groups do not differ by $>1.5$ days, and similarly to reject a difference of $\geq 1.5$ days. ${ }^{6}$ This sample size would also provide similar power with McNemar test to detect a $25 \%$ difference between monitors in the proportion of patients deemed fluid responsive, assuming agreement as low as $57 \%$.

Simple descriptive statistics were used to summarize demographic characteristics of patients. Agreement between the NICOM and EDM was assessed by Bland-Altman plots, correlations between absolute SV values, and McNemar tests comparing the proportion of patients deemed fluid responsive (10\% increase in SV after a fluid challenge) at each time point. At each time point, responses from all fluid challenges were included in the Bland-Altman and McNemar tests. However, to address concern about the effect of correlation of multiple data points per patient in the Bland-Altman analysis, we present prediction intervals, which are based on the number of patients, rather than the usual limits of agreement. (Prediction intervals, calculated as bias $\pm \mathrm{t}_{(0.975}$, ${ }_{n-1)} \times \mathrm{SD} \sqrt{1+(1 / n)}$, were all within $0.3 \mathrm{~mL}$ of their respective limits of agreement.) Differences between phase I and phase II patients in the PACU and in time to return of bowel function and maximum increase in creatinine from baseline, were assessed and described with $t$ tests and confidence limits where appropriate. Because patients having laparoscopic surgery and/or hemicolectomies tend to have a shorter LOS than patients having open procedures or rectal surgery, LOS data were first analyzed with multivariate analysis of variance including these 2 factors and the phase of the study. In the absence of an interaction, difference between groups in total LOS was analyzed with a $t$ test.

Postoperative recovery variables were analyzed with a variety of methods. To assess difference in postoperative renal function, we compared the peak fractional change in creatinine, or the maximum increase in creatinine from baseline, as detailed by Shaw et al. ${ }^{19}$ Logistic regression was used to test the association between study phase and the incidence of fevers, wound dehiscence, reoperation, and need for antibiotics. Linear regression was used to test for association between study phase and postoperative vomiting (number of patients experiencing vomiting), need for antiemetics (number of patients requiring antiemetics), and postoperative oxygen requirement (number of days patients required supplemental oxygen).

\section{RESULTS}

One hundred patients were enrolled in this study. Intraoperative data were available for 49 patients from phase I and 50 patients from phase II, while postoperative data were available for all study participants. One patient in phase I had no useful intraoperative data due to monitor malfunction. Patient demographic information is included in Table 1. The patients were well matched for most demographic characteristics. Of note, phase I contained more patients having laparoscopic surgery than phase II $(P=0.0440)$.

\section{Intraoperative Characteristics}

Intraoperative characteristics of study subjects are presented in Table 1. No significant differences were found in phase I versus phase II patients with regard to anesthesia time, surgery time, volume of crystalloid or blood products used intraoperatively, estimated blood loss, and total dose of analgesic or antiemetic drugs used intraoperatively. After correction for time under 


\begin{tabular}{|c|c|c|}
\hline & Phase I (EDM) & Phase II (NICOM) \\
\hline Age $(y)$ & $53.2 \pm 16.5$ & $57.2 \pm 14.6$ \\
\hline ASA physical status I/II/III & $0 / 15 / 35$ & $0 / 9 / 41$ \\
\hline Body mass index & $28.8 \pm 6.7$ & $28.1 \pm 4.4$ \\
\hline Gender (male/female) & $32(64 \%) / 18(36 \%)$ & $25(50 \%) / 25$ (50\%) \\
\hline Renal disease & $12 / 50(24 \%)$ & $19 / 50(38 \%)$ \\
\hline $\begin{array}{l}\text { Baseline creatinine } \\
(\mathrm{mg} / \mathrm{dL})\end{array}$ & $0.93 \pm 0.20$ & $0.87 \pm 0.21$ \\
\hline Cardiac risk factors & $38 / 50(76 \%)$ & $43 / 50(86 \%)$ \\
\hline Hypertension & $29 / 50(58 \%)$ & $26 / 50(52 \%)$ \\
\hline Obesity & $15 / 50(30 \%)$ & $17 / 50(34 \%)$ \\
\hline Diabetes mellitus & $8 / 50(16 \%)$ & $13 / 50(26 \%)$ \\
\hline Current smoking & $4 / 50(8 \%)$ & $9 / 50(18 \%)$ \\
\hline $\begin{array}{l}\text { Baseline systolic blood } \\
\text { pressure }(\mathrm{mm} \mathrm{Hg})\end{array}$ & $136.3 \pm 20.9$ & $134.5 \pm 20.9$ \\
\hline $\begin{array}{l}\text { Baseline diastolic blood } \\
\text { pressure }(\mathrm{mm} \mathrm{Hg})\end{array}$ & $78.3 \pm 10.0$ & $75.1 \pm 12.3$ \\
\hline Respiratory disease & $20 / 50(40 \%)$ & $21 / 50(42 \%)$ \\
\hline Vascular disease & $6 / 50(12 \%)$ & $8 / 50(16 \%)$ \\
\hline Liver disease & $4 / 50(8 \%)$ & $7 / 50(14 \%)$ \\
\hline \multicolumn{3}{|l|}{ Type of surgery } \\
\hline Laparoscopic* & $33 / 50(66 \%) *$ & $23 / 50(46 \%) *$ \\
\hline Hemicolectomy & $26 / 50(52 \%)$ & $17 / 50$ (34\%) \\
\hline Low anterior resection & $10 / 50(20 \%)$ & $14 / 50(28 \%)$ \\
\hline Small bowel resection & $5 / 50(10 \%)$ & $7 / 50$ (14\%) \\
\hline Abdominoperineal resection & $1 / 50(2 \%)$ & $4 / 50(8 \%)$ \\
\hline Total abdominal colectomy & $3 / 50(6 \%)$ & 4/50 (8\%) \\
\hline $\begin{array}{l}\text { Diverting loop ileostomy } \\
\text { created }\end{array}$ & $10 / 50(20 \%)$ & $15 / 50(30 \%)$ \\
\hline Anesthesia time (min) & $290.9 \pm 136.2$ & $296.6 \pm 119.7$ \\
\hline Surgery time $(\mathrm{min})$ & $246.5 \pm 127.0$ & $244.9 \pm 111.3$ \\
\hline Total Voluven $(\mathrm{mL})^{*}$ & $1076.2 \pm 516.9 *$ & $1370.0 \pm 649.7^{*}$ \\
\hline Total crystalloid (mL) & $2189.7 \pm 1028.5$ & $2213.2 \pm 801.4$ \\
\hline Total blood products (mL) & $17.74 \pm 125.4$ & $75.60 \pm 321.4$ \\
\hline Estimated blood loss (mL) & $235.6 \pm 315.1$ & $290.8 \pm 337.2$ \\
\hline Urine output $(\mathrm{mL})$ & $567.5 \pm 384.6$ & $483.2 \pm 277.1$ \\
\hline Total fentanyl (mg) & $202.5 \pm 75.30$ & $233.0 \pm 94.69$ \\
\hline $\begin{array}{l}\text { Total epidural } \\
\text { bupivacaine (mg) }\end{array}$ & $47.50 \pm 31.29$ & $47.81 \pm 32.66$ \\
\hline $\begin{array}{l}\text { Prebolus mean } \\
\text { arterial blood } \\
\text { pressure }(\mathrm{mm} \mathrm{Hg})^{*}\end{array}$ & $85.6 \pm 14.3 *$ & $82.5 \pm 14.1 *$ \\
\hline $\begin{array}{l}\text { Prebolus cardiac index } \\
\qquad\left(\mathrm{L} / \mathrm{min} / \mathrm{m}^{2}\right)^{*}\end{array}$ & $3.8 \pm 1.8^{*}$ & $3.3 \pm 2.2 *$ \\
\hline Prebolus stroke volume $(\mathrm{mL}) \dagger$ & $99.4 \pm 28.6 \dagger$ & $89.9 \pm 24.3 \dagger$ \\
\hline Required phenylephrine & $30 / 50(60 \%)$ & $36 / 50(72 \%)$ \\
\hline Required ephedrine & $16 / 50(32 \%)$ & $24 / 50$ (48\%) \\
\hline Required a vasodilator & $12 / 50(24 \%)$ & $11 / 50(22 \%)$ \\
\hline
\end{tabular}

Measurements are either $n(\%)$ or mean \pm SD.

$\mathrm{EDM}=$ esophageal Doppler monitor; NICOM = noninvasive cardiac output monitor.

$* P<0.05$

$\dagger P<0.001$; otherwise there were no significant differences between groups.

anesthesia, patients in phase I received approximately $273 \mathrm{~mL}$ less colloid than patients in phase II $(P=0.0005)$. No significant differences were observed in the number of patients in each phase requiring intraoperative phenylephrine $(P=0.2053)$, ephedrine $(P=0.1025)$, or vasodilators $(P=0.8122)$.

\section{Agreement Between the NICOM and EDM}

The mean baseline SV was $94.10 \pm 26.91 \mathrm{~mL}$ for the NICOM and $84.48 \pm 30.38 \mathrm{~mL}$ for the EDM. There was a consistent and significant correlation of baseline SV between monitors (Pearson correlation coefficient, 0.45 ; lower 95\% confidence limit $=0.30 ; P<0.0001)$. In addition, Bland-Altman analyses were performed at baseline and at 5, 10, and 15 minutes after the fluid boluses (Fig. 1, A-D). At baseline, bias was $-2.15 \mathrm{~mL}$, precision was $31.73 \mathrm{~mL}$, and the prediction interval extended from -65.4 to $61.1 \mathrm{~mL}$. At $5 \mathrm{~min}-$ utes, bias was $-4.83 \mathrm{~mL}$, precision was $33.76 \mathrm{~mL}$, and the prediction interval extended from -72.2 to $62.5 \mathrm{~mL}$. At 10 minutes, bias was $-4.52 \mathrm{~mL}$, precision was $33.34 \mathrm{~mL}$, and the prediction interval extended from -71.0 to $62.0 \mathrm{~mL}$. At 15 minutes, bias was $-3.66 \mathrm{~mL}$, precision was $34.57 \mathrm{~mL}$, and the prediction interval extended from -72.6 to 65.3 $\mathrm{mL}$.

The level of agreement on fluid responsiveness between the monitors after fluid challenge is shown in Table 2. Using a $10 \%$ increase in SV after fluid challenge, agreement between the 2 monitors at 5 minutes was observed at $60 \%$ and estimated to be at least $54 \%$. At 10 minutes, agreement was observed at $61 \%$ and estimated to be at least $56 \%$. At 15 minutes, agreement was observed at $66 \%$ and estimated to be at least $61 \%$. No statistically significant systematic disagreement was found between the monitors at any time point $(\mathrm{McNemar}$ statistic $=0.0286-0.6279, P>0.05$ at all time points) At the higher threshold for fluid responsiveness of a $20 \%$ increase in SV, the observed 15-minute agreement between the monitors increased from $66 \%$ to $76 \%$ (lower $95 \%$ confidence limits of $61 \%$ and $71 \%$, respectively), with no substantial change in the $\chi^{2}$ values or the McNemar statistics (Table 2).

The EDM had significantly more missing hemodynamic data than the NICOM at all time points $(4.6 \%$ vs $2.1 \%$ at baseline, $6.0 \%$ vs $2.5 \%$ at 5 minutes, $9.4 \%$ vs $3.7 \%$ at $10 \mathrm{~min}-$ utes, and $10.1 \%$ vs $5 \%$ at 15 minutes; $P<0.0001$ at all time points). Between $61 \%$ and $81 \%$ of the data that were missing on the EDM were available on the NICOM.

\section{Postoperative Recovery}

There was no significant difference between patients with regard to time spent in the PACU, amount of IV fluids required, maximum nausea/pain scores, or the number of patients requiring antiemetics, analgesics, inotropes, or vasodilators (Table 3).

Similarly, no significant difference in total LOS was found between the 2 groups. The mean LOS was $6.56 \pm 4.32$ days in phase I and $6.07 \pm 2.85$ days in phase II, and $95 \%$ confidence limits for the difference were -0.96 to +1.95 days $(P=0.5016)$. Patients in phase II had a significantly shorter time to bowel sounds (mean difference $=9.66 \pm 17.66$ hours, $P=0.0114$ ), but a longer time to first oral liquids (mean difference $=7.28 \pm 15.62$ hours, $P=0.0228$ ). However, there was no difference in time to solid intake, first flatus, first bowel movement, incidence of acute kidney injury, or other complications. (Table 4).

\section{DISCUSSION}

The NICOM performed similarly to the EDM when used for GDFT. There was reasonable correlation of baseline SV, and agreement on fluid responsiveness between the monitors was observed at $66 \%$ and estimated to be at least $61 \%$ at 15 minutes with no significant systematic disagreement at any time point. Moreover, phase I and phase II patients had similar clinical outcomes, with no clinically significant 
A

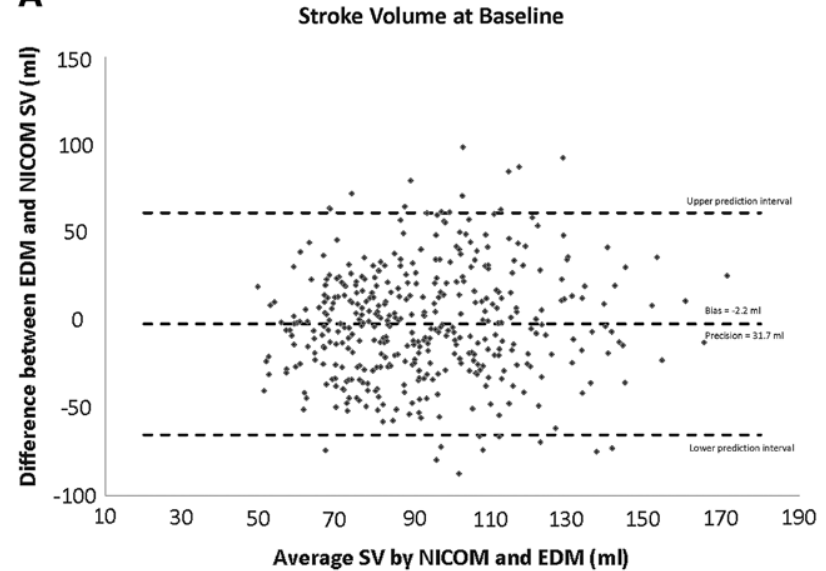

C

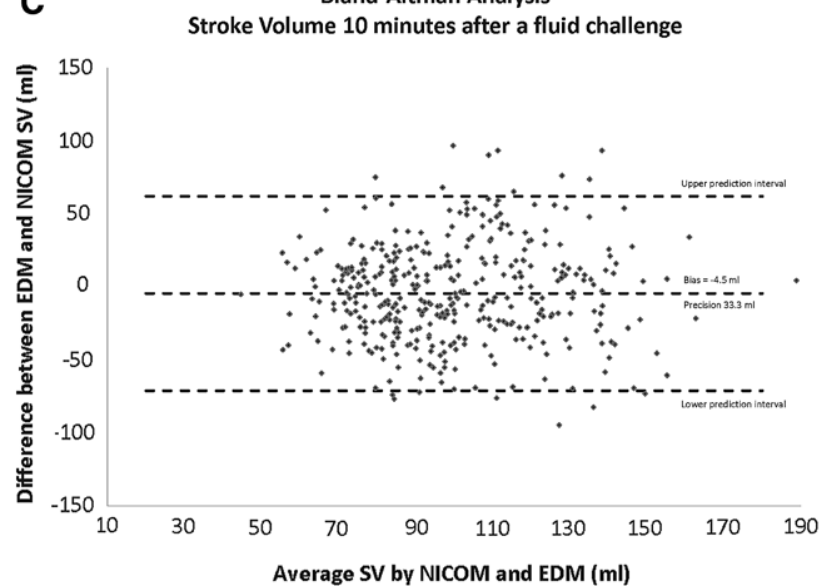

B Bland-Altman Analysis

Stroke Volume 5 minutes after fluid challenge

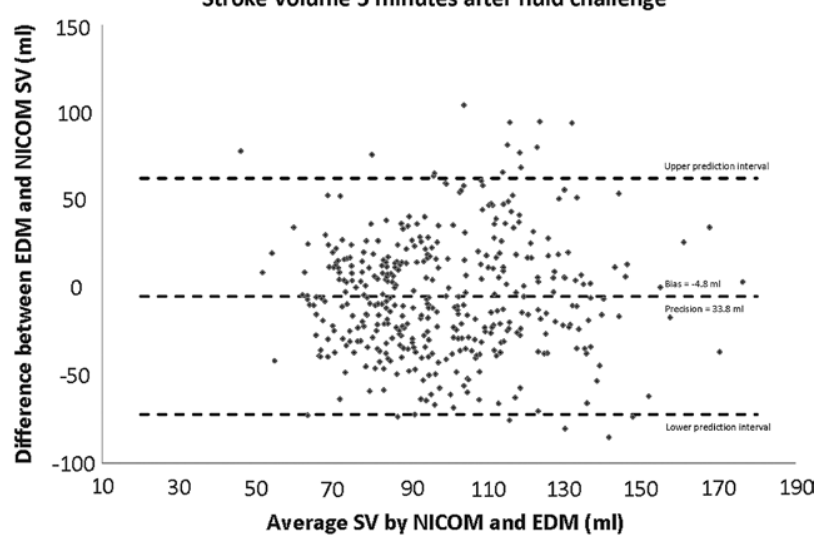

Bland-Altman Analysis
Stroke Volume 15 minutes after a fluid challenge

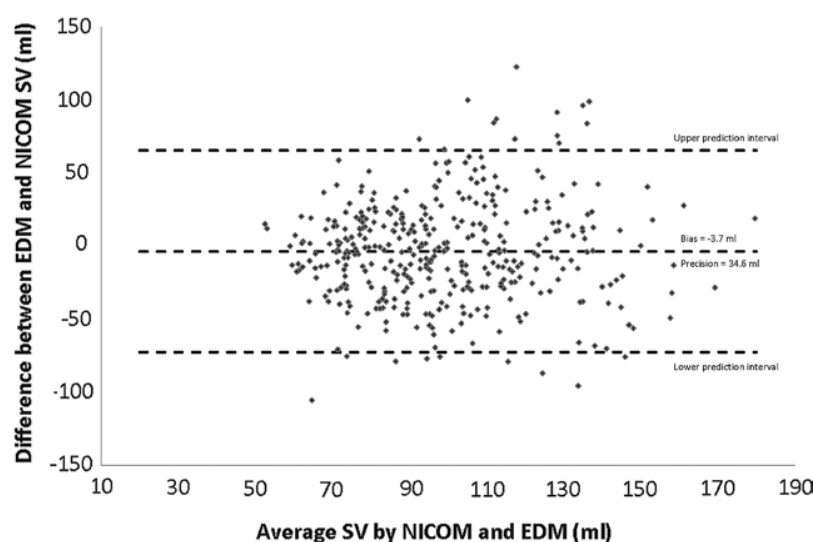

Figure 1. A, Baseline Bland-Altman analysis at baseline. Difference (bias) between monitors is calculated as esophageal Doppler monitor (EDM) minus noninvasive cardiac output monitor (NICOM); negative bias indicates EDM lower. Percentage error $(1.98 \times$ precision/mean stroke volume [SV] at baseline $)=67.7 \%$. The analysis includes 412 simultaneous observations. Prediction intervals reflect the 99 patients measured and are within $0.3 \mathrm{~mL}$ of the limits of agreement. B, Baseline Bland-Altman analysis at 5 minutes. Difference (bias) between monitors is calculated as EDM minus NICOM; negative bias indicates EDM lower. Percentage error $(1.98 \times$ precision $/$ mean SV at 5 minutes $)=67.7 \%$. The analysis includes 405 simultaneous observations. Prediction intervals reflect the 99 patients measured and are within $0.3 \mathrm{~mL}$ of the limits of agreement. C, Baseline Bland-Altman analysis at 10 minutes. Difference (bias) between monitors is calculated as EDM minus NICOM; negative bias indicates EDM lower. Percentage error $(1.98 \times$ precision/mean SV at 10 minutes $)=66.4 \%$. The analysis includes 391 simultaneous observations. Prediction intervals reflect the 99 patients measured and are within $0.3 \mathrm{~mL}$ of the limits of agreement. D, Baseline Bland-Altman analysis at 15 minutes. Difference (bias) between monitors is calculated as EDM minus NICOM; negative bias indicates EDM lower. Percentage error $(1.98 \times$ precision/mean SV at 15 minutes $)=69.3 \%$. The analysis includes 388 simultaneous observations. Prediction intervals reflect the 99 patients measured and are within $0.3 \mathrm{~mL}$ of the limits of agreement.

\begin{tabular}{|c|c|c|c|c|}
\hline Time point & $\begin{array}{l}\text { NICOM } n(\%) \\
\text { fluid responsive }\end{array}$ & $\begin{array}{l}\text { Esophageal Doppler monitor } n(\%) \\
\text { fluid responsive }\end{array}$ & $\begin{array}{c}\text { Agreement } \\
\text { (95\% confidence limits)* }\end{array}$ & $\begin{array}{l}\text { McNema } \\
P \text { value } \dagger\end{array}$ \\
\hline \multicolumn{5}{|l|}{$10 \%$ increase in SV } \\
\hline 5 min postbolus & 155/393 (39.4) & $146 / 393(37.2)$ & $59.5 \%(54.5-64.4)$ & 0.4754 \\
\hline 10 min postbolus & $154 / 377(40.8)$ & $145 / 377(38.5)$ & $61 \%(55.9-66.0)$ & 0.4579 \\
\hline 15 min postbolus & $148 / 375(39.5)$ & $157 / 375(41.9)$ & $65.6 \%(60.6-70.4)$ & 0.4281 \\
\hline Fluid responsive overall & $206 / 368(56.0)$ & $208 / 368(56.5)$ & $62 \%(56.8-66.9)$ & 0.8658 \\
\hline \multicolumn{5}{|l|}{$20 \%$ increase in SV } \\
\hline 5 min postbolus & 76/393 (19.3) & 78/393 (19.8) & $74 \%(69.4-78.3)$ & 0.8430 \\
\hline 10 min postbolus & $74 / 377$ (19.6) & $75 / 377$ (19.9) & $71.1 \%(66.2-75.6)$ & 0.9237 \\
\hline 15 min postbolus & $75 / 375(20.0)$ & $87 / 375(23.2)$ & $76 \%(71.4-80.2)$ & 0.2059 \\
\hline Fluid responsive overall & $108 / 359(30.1)$ & $124 / 359(34.5)$ & $66 \%(60.9-70.9)$ & 0.1475 \\
\hline
\end{tabular}

Denominator for fluid-responsive percent shows total number of responses measured with both techniques simultaneously.

$\mathrm{NICOM}=$ noninvasive cardiac output monitor; SV = stroke volume.

*Percent of responses in agreement, with Clopper-Pearson (exact) $95 \%$ confidence limits.

$\dagger$ McNemar $P$ value $>0.05$ indicates the absence of significant systematic disagreement between 2 techniques. 


\begin{tabular}{lccc}
\hline Table 3. Patient Characteristics in Postanesthesia Care Unit & Phase II & P value \\
& Phase I & $239.9 \pm 107.6$ & 0.9294 \\
Time spent (min) & $241.8 \pm 105.4$ & $398.0 \pm 567.0$ & 0.5177 \\
Total crystalloid $(\mathrm{mL})$ & $335.5 \pm 366.9$ & $91.2 \pm 243.3$ & 0.7367 \\
Total colloid used $(\mathrm{mL})$ & $107.0 \pm 222.5$ & $1.50 \pm 2.74$ & 0.2476 \\
Maximum nausea & $0.95 \pm 1.82$ & $4.56 \pm 3.12$ & 0.3458 \\
Maximum pain at rest & $3.94 \pm 3.12$ & $5.57 \pm 3.65$ & 0.9083 \\
Maximum pain with activity & $5.66 \pm 3.57$ & $17 / 49(35 \%)$ & 0.2424 \\
Required antiemetics & $12 / 50(24 \%)$ & $24 / 49(49 \%)$ & 0.7638 \\
Required analgesic medications & $26 / 50(52 \%)$ & $2 / 49(4 \%)$ & 0.1490 \\
Required an inotrope & $0 / 50(0 \%)$ & $5 / 49(10 \%)$ & 0.4429 \\
Required a vasodilator & $3 / 50(6 \%)$ & & \\
\hline
\end{tabular}

Measurements are mean \pm SD or $n(\%)$. Two-sample $t$ test or $\chi^{2}$ test used to detect differences between the 2 groups. One patient in phase II had missing postanesthesia care unit data.

\begin{tabular}{|c|c|c|c|}
\hline & Phase I & Phase II & $P$ value \\
\hline Total length of stay $(\mathrm{h})$ & $157.5 \pm 103.6$ & $145.7 \pm 68.3$ & 0.5016 \\
\hline Time to first bowel sounds (h) & $20.72 \pm 14.78$ & $11.06 \pm 19.75$ & 0.0114 \\
\hline Time to first flatus $(\mathrm{h})$ & $44.15 \pm 26.17$ & $41.98 \pm 35.13$ & 0.7595 \\
\hline Time to first bowel movement ( $h$ ) & $64.30 \pm 40.59$ & $50.16 \pm 44.19$ & 0.1387 \\
\hline Time to first liquid $(\mathrm{h})$ & $9.89 \pm 7.67$ & $17.17 \pm 20.62$ & 0.0228 \\
\hline Time to first solid (h) & $52.34 \pm 50.47$ & $50.67 \pm 42.98$ & 0.8614 \\
\hline Incidence of fever & $17 / 50(34 \%)$ & $14 / 50(28 \%)$ & 0.5171 \\
\hline Patients required postoperative antibiotics & $10 / 50(20 \%)$ & $7 / 50(14 \%)$ & 0.4266 \\
\hline Hypoxia requiring oxygen therapy (total episodes) & 22 & 41 & 0.1339 \\
\hline Maximum \% change in creatinine from baseline & $28.75 \pm 48.07$ & $22.89 \pm 22.84$ & 0.4470 \\
\hline Incidence of wound dehiscence & $2 / 50(4 \%)$ & $3 / 50(6 \%)$ & 0.6485 \\
\hline Incidence of reoperation & $2 / 50(4 \%)$ & $0 / 50(0 \%)$ & 0.9426 \\
\hline Vomiting, number of patients (\%) & $23 / 50(46 \%)$ & $23 / 50(46 \%)$ & 1.000 \\
\hline Antiemetic therapy, number of patients (\%) & $35 / 50(70 \%)$ & $39 / 50(78 \%)$ & 0.3618 \\
\hline Postdischarge emergency department visit & $7 / 50$ (14\%) & $8 / 50(16 \%)$ & 0.7794 \\
\hline
\end{tabular}

Measurements are mean \pm SD or $n(\%)$. Two-sample $t$ test or $\chi^{2}$ test used to detect differences between the 2 groups.

differences in LOS, postoperative bowel recovery, renal function, or other complications.

The esophageal Doppler is a thin, flexible probe inserted into a patient's midesophagus where it measures blood flow velocity in the descending thoracic aorta. It uses a nomogram to calculate aortic cross-sectional area based on the patient's height, weight, and age..$^{20}$ The EDM is a valid measure of $\mathrm{CO}$ when compared with a pulmonary artery catheter ${ }^{21}$ and is also appropriate for measuring hemodynamic response to fluid boluses in GDFT. ${ }^{4}$ However, experience is required to become skilled in using this device, and an optimal signal may be difficult to obtain. ${ }^{12}$

Transthoracic bioimpedance technology is used to measure changes in amplitude of high-frequency waves transmitted across the thorax. It has been available as a measure of $\mathrm{CO}$ for approximately 20 years, but has not been widely used due to problems with electrical interference and because the algorithm relies on hemodynamic stability and the absence of cardiac dysrhythmias. ${ }^{12}$ The NICOM uses an improved algorithm based on bioreactance, which measures phase shifts in high-frequency waves transmitted across the thorax, with a nearly 100 -fold improvement in the signal-to-noise ratio. ${ }^{15}$ The device consists of 4 pads placed across the thorax that simultaneously emit and detect high-frequency, low-amplitude electrical currents and are connected to the monitor via a single cable. The
NICOM has been validated for clinically acceptable precision and accuracy when compared with the pulmonary artery catheter in critically ill postoperative cardiothoracic surgery patients. . $^{15,22}$

GDFT is a means of optimizing intravascular volume status and tissue perfusion and has been associated with shorter hospital LOS, fewer intensive care unit admissions, earlier return of bowel function, and less postoperative nausea/vomiting. ${ }^{5-10}$ Systematic reviews have also reported decreased rates of acute renal injury ${ }^{23}$ as well as a decrease in minor and major gastrointestinal complications ${ }^{24}$ in patients receiving perioperative GDFT. Interestingly, a recent meta-analysis found a highly significant reduction in morbidity in patients receiving GDFT regardless of the monitoring technique used, that is, pulmonary artery catheter, esophageal Doppler, or other method. ${ }^{25}$ Indeed, the benefits of GDFT are likely not monitor specific, but rather result from a systematic approach to hemodynamic optimization. ${ }^{26}$

Emerging CO monitors are typically validated by comparison to a reference standard, often pulmonary artery catheter-based thermodilution, which many consider to be the "gold standard" for $\mathrm{CO}$ measurement. Interestingly, the true gold standard for CO measurement is a surgically implanted aortic flowprobe, ${ }^{27}$ which the NICOM has been validated against in animal studies with clinically 
acceptable accuracy. ${ }^{28}$ However, pulmonary artery catheter-based thermodilution techniques are used as the practical gold standard for human CO measurement, despite being an imperfect reference. ${ }^{27,29}$ Often used to compare $\mathrm{CO}$ monitors, Bland-Altman analysis assesses agreement between 2 methods by measuring systematic differences between methods (bias) and standard deviation of these differences (precision), with the prediction interval calculated as bias $\pm(1.98 \times$ precision $) .{ }^{30}$ In addition, the percentage error (1.98 $\times$ precision/mean CO or SV at a given time) is often reported, and it has been proposed that the threshold for accepting a new $\mathrm{CO}$ monitor should be a percentage error $<30 \%$ when compared with thermodilution. ${ }^{31}$ Interestingly, a subsequent meta-analysis found that none of 4 commonly used minimally invasive $\mathrm{CO}$ monitoring techniques (pulse contour analysis, esophageal Doppler, partial $\mathrm{CO}_{2}$ rebreathing, and thoracic bioimpedance) have $<30 \%$ percentage error, and most techniques approach $45 \%$ percentage error when compared with thermodilution. ${ }^{32} \mathrm{~A}$ more recent study measured cardiac index by esophageal Doppler, LiDCO Rapid (LiDCO LTD, London, UK), and the FloTrac (Edwards Lifesciences, Irvine, CA) side by side in cardiac surgery patients and found a percentage error of $64.5 \%, 54.2 \%$, and $47.6 \%$, respectively, when compared with thermodilution. Moreover, the same study found relatively poor agreement between the monitors and thermodilution regarding whether $\mathrm{CO}$ increased after fluid bolus (63.6\% agreement for esophageal Doppler, 54.6\% agreement for LiDCO, and 33.3\% agreement for FloTrac). ${ }^{33}$ Taken as a whole, these data suggest at least moderate discord between each method and the reference, thermodilution. This is not surprising given the different methods involved and the inherent error of thermodilution $(10 \%-$ $20 \%$ ). As such, the quest for close to complete agreement between 2 methods may be illusive. However, the acceptable limits of agreement between 2 minimally invasive CO monitors have not been ascertained.

Bland-Altman analysis has many limitations. Most notably, it evaluates the ability of 2 technologies to measure the same quantity under stable conditions. However, many authors agree that the most important characteristic of a CO monitor is its ability to track changes in $\mathrm{CO} \cdot{ }^{32,34,35}$ Similarly, the focus of our study was not the absolute agreement between the NICOM and EDM, but rather their ability to measure SV response to fluid bolus. To that end, the NICOM performed similarly to the EDM. Using a $10 \%$ increase in SV, the 2 monitors declared a similar proportion of patients as fluid responsive at each time point (e.g., $39.5 \%$ fluid responsive vs $41.9 \%$ at 15 minutes), with no significant systematic disagreement and an observed agreement of $66 \%$ at 15 minutes.

As noted previously, 12,13 the EDM has many limitations. The device is somewhat invasive, requiring placement of a tube in the esophagus, and is not well tolerated by awake patients. In addition, some training is required to achieve competency, and an optimal signal can be difficult to obtain in some patients. Moreover, electrocautery interferes with the EDM signal, resulting in data loss during surgery. In our analysis, there was a significantly higher proportion of missing data using the EDM, with the majority of that being available by the NICOM, indicating that the NICOM may be more reliable for GDFT in the perioperative environment. We postulate that these missing data are due to interference from electrocautery and that the signal averaging of the NICOM renders it more resistant to electrocautery than the EDM.

Few clinically significant differences in recovery were observed between patients whose GDFT was guided by the NICOM versus the EDM. Patients in phase II not only received significantly more colloid intraoperatively, but also tended to have a lower mean arterial BP, cardiac index, and SV than patients in phase I, which may account for this difference. In addition, all recovery variables in the PACU were similar, including LOS, volume of IV fluids, requirements for vasoactive drugs, and relevant measures of pain and nausea.

Also, few significant differences between groups were found in postoperative recovery. No significant differences in total LOS or recovery of bowel or renal function were noted. Moreover, there were no significant differences between groups in postoperative surgical, pulmonary, or infectious complications. The total mean LOS (phase 1: $6.56 \pm 4.32$ days, phase II: $6.07 \pm 2.85$ days) was similar to that seen in a previous GDFT study at our institution. ${ }^{6}$ Patients in phase II had a significantly shorter time to first bowel sounds but a longer time to first liquid intake, with no significant differences in time to solid intake, first bowel movement, postoperative nausea, vomiting, or antiemetic treatment. It is interesting to note that although bowel sounds are considered a routine part of the abdominal examination, their diagnostic value has been called into question, ${ }^{36}$ particularly in postoperative patients. ${ }^{37}$

This study had some limitations. Patients were assigned to groups sequentially rather than by randomization. We chose this approach so that agreement statistics between the NICOM and EDM could be calculated after phase I before proceeding to phase II. This allowed us to verify the accuracy of the NICOM before instituting it into our clinical algorithm, as this was the first study that used the NICOM to guide GDFT. There was a relatively frequent incidence of laparoscopy in our study, which can induce a number of hemodynamic changes including alterations in venous return and regional blood flow, decreased SV and CO, and increases in afterload and systemic vascular resistance. ${ }^{38}$ In addition, more phase I patients had laparoscopic surgery and hemicolectomies compared with phase II patients. However, there was no interaction between phase of study and type of surgery in the LOS analysis $(P=0.1952$ for laparoscopic $\times$ phase interaction, $P=0.1519$ for hemicolectomy $\times$ phase interaction). Alterations in venous pressure during pneumoperitoneum may render pressure-based measures of preload less accurate, ${ }^{39}$ thus increasing the need for noninvasive technologies to measure hemodynamics during laparoscopy, such as the NICOM. It is interesting to note that although the EDM algorithm makes assumptions about regional blood flow, which may be altered by pneumoperitoneum, the EDM and NICOM performed similarly in our analysis. In addition, our baseline EDM and NICOM SV measurements showed only a modest correlation (Pearson correlation coefficient, 0.45; lower 95\% confidence limit $=0.30 ; P<0.0001$ ), and the Bland-Altman 
limits of agreement/prediction intervals were large relative to the mean SV. However, we believe that this reflects the inherent imprecision of the comparator (EDM) in this study. Moreover, given the lack of significant differences in clinical outcome between the NICOM and EDM groups, we maintain that the 2 monitors perform similarly for clinical use as part of a GDFT algorithm.

It also should be noted that the ERAS protocol at our institution is a broad-based protocol with many components. We believe it is likely that each component contributes in some way to the overall benefit patients receive. However, it would be quite difficult to show that any additional intervention (such as NICOM or EDM) provides any additional benefit while studying them in the context of our ERAS protocol. Although the data were collected by independent research staff not involved in the intraoperative management of patients, it was not possible to blind the anesthesiologists to the treatment group. However, in both phases of the study, fluid was administered according to a protocol, which may have minimized bias. Also, some of the medications used intraoperatively (epidural local anesthetics, opioids) may have affected bowel function, although there were no significant differences in intraoperative opioid or local anesthetic use. Finally, since this study was conducted over a period of approximately 11 months, it is feasible that surgical skills and/or technology improved during that time. However, GDFT is routinely practiced in this cohort of surgical patients.

In conclusion, no significant differences were found between the NICOM and EDM in ability to assess fluid responsiveness as part of a GDFT algorithm. In addition, there were no clinically significant differences in LOS and postoperative recovery when GDFT was guided by the NICOM or the EDM. However, the NICOM captured significantly more data than the EDM, was more user friendly, and is less invasive than the EDM. Therefore, NICOM may be a viable alternative for guiding GDFT.

\section{APPENDIX 1: DUKE ENHANCED RECOVERY AFTER SURGERY PROTOCOL}

\section{Surgical Planning/Preoperative Screening Clinic}

- Identify elective surgery patients who can benefit from participation

- Educate patients about the track and our expectations
- Reinforce with a written copy of our plan and expectations

- Screen for malnutrition, tobacco abuse, and diabetes

\section{Preoperative Screening Clinic}

- Routine preoperative screening, specific attention to known risk factors

- Distribute

Nutritional supplements if serum albumin is $<3.5 \mathrm{~g} / \mathrm{dL}$ Smoking cessation information

Chlorhexidine sponges for 2 preoperative showers

Carbohydrate Drink for Morning of Surgery

- Reinforce with written instructions and contact information

\section{Preoperative Holding, Day of Operation}

- Identify ERAS patients and initiate protocol

- Epidural anesthesia placement -T8-T12 region

- Thromboprophylaxis with heparin 5000 U subcutaneous after placement of epidural

\section{Intraoperative}

- Antibiotic prophylaxis before incision

- Sequential compression devices placed before induction of anesthesia

- Use epidural throughout case without any IV opioids

- Goal-directed IV fluid therapy

- Orogastric tube removed before leaving OR

- Foley discontinued in OR, except for pelvic operations

\section{Postoperative}

- Identify Enhanced Recovery After Surgery patients for protocol participation

- Diet begins night of surgery

- Ambulation begins night of surgery

- Head of bed at $30^{\circ}$ at all times

- Less than/equal to 1 L IV fluids over 24 hours (70 kg)

- Epidural and SCD continuation

- Postoperative thromboprophylaxis begins postoperative day 1

- Close blood glucose monitoring and maintain normoglycemia 


\section{APPENDIX 2: GOAL-DIRECTED FLUID THERAPY PROTOCOL}

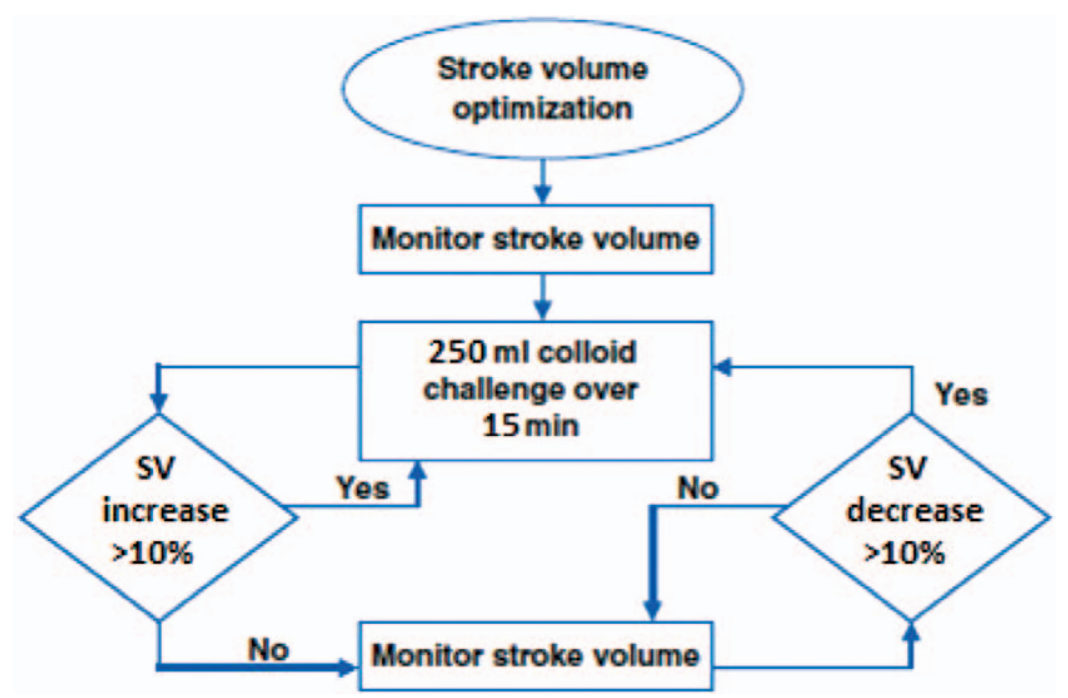

\section{DISCLOSURES}

Name: Nathan H. Waldron, MD.

Contribution: This author helped design the study, conduct the study, analyze the data, and write the manuscript.

Attestation: Nathan H. Waldron has seen the original study data, reviewed the analysis of the data, and approved the final manuscript.

Conflicts of Interest: The author has no conflicts of interest to declare.

Name: Timothy E. Miller, MB ChB, FRCA.

Contribution: This author helped design the study, conduct the study, analyze the data, and write the manuscript.

Attestation: Timothy E. Miller has seen the original study data, reviewed the analysis of the data, and approved the final manuscript.

Conflicts of Interest: The author has no conflicts of interest to declare.

Name: Julie K. Thacker, MD.

Contribution: This author helped conduct the study and write the manuscript.

Attestation: Julie K. Thacker reviewed the analysis of the data and approved the final manuscript.

Conflicts of Interest: The author has no conflicts of interest to declare.

Name: Amy K. Manchester, MD.

Contribution: This author helped conduct the study and write the manuscript.

Attestation: Amy K. Manchester has seen the original study data and approved the final manuscript.

Conflicts of Interest: The author has no conflicts of interest to declare.

Name: William D. White, MPH.

Contribution: This author helped design the study, analyze the data, and write the manuscript.

Attestation: William D. White has seen the original study data, reviewed the analysis of the data, and approved the final manuscript.

Conflicts of Interest: The author has no conflicts of interest to declare.
Name: John Nardiello, MD.

Contribution: This author helped conduct the study and write the manuscript.

Attestation: John Nardiello has seen the original study data and approved the final manuscript.

Conflicts of Interest: The author has no conflicts of interest to declare.

Name: Magdi A. Elgasim, MBBS.

Contribution: This author helped conduct the study.

Attestation: Magdi A. Elgasim has seen the original study data and approved the final manuscript.

Conflicts of Interest: The author has no conflicts of interest to declare.

Name: Richard E. Moon, MD, CM, MSc, FRCP (C), FACP, FCCP. Contribution: This author helped design the study, conduct the study, and write the manuscript.

Attestation: Richard E. Moon has seen the original study data and approved the final manuscript.

Conflicts of Interest: The author has no conflicts of interest to declare.

Name: Tong J. Gan, MD, MHS, FRCA, Li Ac.

Contribution: This author helped design the study, conduct the study, analyze the data, and write the manuscript.

Attestation: Tong J. Gan has seen the original study data, reviewed the analysis of the data, approved the final manuscript, and is the author responsible for archiving the study files.

Conflicts of Interest: Tong J. Gan serves as a consultant for Baxter, Edwards Life Science, Hospira, and QRx and has received research support from AcelRx, Cheetah, Covidien, Cubist, Deltex, Fresenius, Merck, Pacira, and Premier.

This manuscript was handled by: Maxime Cannesson, MD, PhD.

\section{REFERENCES}

1. Chappell D, Jacob M, Hofmann-Kiefer K, Conzen P, Rehm M. A rational approach to perioperative fluid management. Anesthesiology 2008;109:723-40

2. Bundgaard-Nielsen $M$, Holte $K$, Secher $N H$, Kehlet $H$. Monitoring of peri-operative fluid administration by individualized goal-directed therapy. Acta Anaesthesiol Scand 2007;51:331-40 
3. Kehlet H, Bundgaard-Nielsen M. Goal-directed perioperative fluid management: why, when, and how? Anesthesiology 2009;110:453-5

4. Roche AM, Miller TE, Gan TJ. Goal-directed fluid management with trans-oesophageal Doppler. Best Pract Res Clin Anaesthesiol 2009;23:327-34

5. Abbas SM, Hill AG. Systematic review of the literature for the use of oesophageal Doppler monitor for fluid replacement in major abdominal surgery. Anaesthesia 2008;63:44-51

6. Gan TJ, Soppitt A, Maroof M, el-Moalem H, Robertson KM, Moretti E, Dwane P, Glass PS. Goal-directed intraoperative fluid administration reduces length of hospital stay after major surgery. Anesthesiology 2002;97:820-6

7. Grocott MP, Mythen MG, Gan TJ. Perioperative fluid management and clinical outcomes in adults. Anesth Analg 2005;100:1093-106

8. Mythen MG, Webb AR. Intra-operative gut mucosal hypoperfusion is associated with increased post-operative complications and cost. Intensive Care Med 1994;20:99-104

9. Sinclair S, James S, Singer M. Intraoperative intravascular volume optimisation and length of hospital stay after repair of proximal femoral fracture: randomised controlled trial. BMJ 1997;315:909-12

10. Venn R, Steele A, Richardson P, Poloniecki J, Grounds M, Newman P. Randomized controlled trial to investigate influence of the fluid challenge on duration of hospital stay and perioperative morbidity in patients with hip fractures. Br J Anaesth 2002;88:65-71

11. Bowdle TA. Complications of invasive monitoring. Anesthesiol Clin North America 2002;20:571-88

12. Funk DJ, Moretti EW, Gan TJ. Minimally invasive cardiac output monitoring in the perioperative setting. Anesth Analg 2009;108:887-97

13. Mowatt G, Houston G, Hernandez R, de Verteuil R, Fraser C, Cuthbertson B, Vale L. Systematic review of the clinical effectiveness and cost-effectiveness of oesophageal Doppler monitoring in critically ill and high-risk surgical patients. Health Technol Assess 2009;13:iii-iv, ix-xii, 1-95

14. Raval NY, Squara P, Cleman M, Yalamanchili K, Winklmaier M, Burkhoff D. Multicenter evaluation of noninvasive cardiac output measurement by bioreactance technique. J Clin Monit Comput 2008;22:113-9

15. Squara P, Denjean D, Estagnasie P, Brusset A, Dib JC, Dubois C. Noninvasive cardiac output monitoring (NICOM): a clinical validation. Intensive Care Med 2007;33:1191-4

16. Lassen K, Soop M, Nygren J, Cox PB, Hendry PO, Spies C, von Meyenfeldt MF, Fearon KC, Revhaug A, NordervalS, Ljungqvist $\mathrm{O}$, Lobo DN, Dejong CH; Enhanced Recovery After Surgery (ERAS) Group. Consensus review of optimal perioperative care in colorectal surgery: Enhanced Recovery After Surgery (ERAS) Group recommendations. Arch Surg 2009;144:961-9

17. Ramírez JM, Blasco JA, Roig JV, Maeso-Martínez S, Casal JE, Esteban F, Lic DC; Spanish working group on fast track surgery. Enhanced recovery in colorectal surgery: a multicentre study. BMC Surg 2011;11:9

18. Spanjersberg WR, Reurings J, Keus F, van Laarhoven CJ. Fast track surgery versus conventional recovery strategies for colorectal surgery. Cochrane Database Syst Rev 2011;2:CD007635

19. Shaw AD, Stafford-Smith M, White WD, Phillips-Bute B, Swaminathan M, Milano C, Welsby IJ, Aronson S, Mathew JP, Peterson ED, Newman MF. The effect of aprotinin on outcome after coronary-artery bypass grafting. N Engl J Med 2008;358:784-93
20. Singer M. Esophageal Doppler monitoring of aortic blood flow: beat-by-beat cardiac output monitoring. Int Anesthesiol Clin 1993;31:99-125

21. Dark PM, Singer M. The validity of trans-esophageal Doppler ultrasonography as a measure of cardiac output in critically ill adults. Intensive Care Med 2004;30:2060-6

22. Marqué S, Cariou A, Chiche JD, Squara P. Comparison between Flotrac-Vigileo and Bioreactance, a totally noninvasive method for cardiac output monitoring. Crit Care 2009;13:R73

23. Brienza N, Giglio MT, Marucci M, Fiore T. Does perioperative hemodynamic optimization protect renal function in surgical patients? A meta-analytic study. Crit Care Med 2009;37:2079-90

24. Giglio MT, Marucci M, Testini M, Brienza N. Goal-directed haemodynamic therapy and gastrointestinal complications in major surgery: a meta-analysis of randomized controlled trials. Br J Anaesth 2009;103:637-46

25. Hamilton MA, Cecconi M, Rhodes A. A systematic review and meta-analysis on the use of preemptive hemodynamic intervention to improve postoperative outcomes in moderate and high-risk surgical patients. Anesth Analg 2011;112:1392-402

26. Miller TE, Roche AM, Gan TJ. Poor adoption of hemodynamic optimization during major surgery: are we practicing substandard care? Anesth Analg 2011;112:1274-6

27. Critchley LA, Lee A, Ho AM. A critical review of the ability of continuous cardiac output monitors to measure trends in cardiac output. Anesth Analg 2010;111:1180-92

28. Heerdt PM, Wagner CL, DeMais M, Savarese JJ. Noninvasive cardiac output monitoring with bioreactance as an alternative to invasive instrumentation for preclinical drug evaluation in beagles. J Pharmacol Toxicol Methods 2011;64:111-8

29. Pugsley J, Lerner AB. Cardiac output monitoring: is there a gold standard and how do the newer technologies compare? Semin Cardiothorac Vasc Anesth 2010;14:274-82

30. Bland JM, Altman DG. Statistical methods for assessing agreement between two methods of clinical measurement. Lancet 1986;1:307-10

31. Critchley LA, Critchley JA. A meta-analysis of studies using bias and precision statistics to compare cardiac output measurement techniques. J Clin Monit Comput 1999;15:85-91

32. Peyton PJ, Chong SW. Minimally invasive measurement of cardiac output during surgery and critical care: a meta-analysis of accuracy and precision. Anesthesiology 2010;113:1220-35

33. Phan TD, Kluger R, Wan C, Wong D, Padayachee A. A comparison of three minimally invasive cardiac output devices with thermodilution in elective cardiac surgery. Anaesth Intensive Care 2011;39:1014-21

34. Chikhani M, Moppett IK. Minimally invasive cardiac output monitoring: what evidence do we need? Br J Anaesth 2011;106:451-3

35. Linton NW, Linton RA. Is comparison of changes in cardiac output, assessed by different methods, better than only comparing cardiac output to the reference method? Br J Anaesth 2002;89:336-7

36. Harris S, Naina HV, Kuppachi S. Look, feel, listen or look, listen, feel? Am J Med 2007;120:e3

37. West M, Klein MD. Is abdominal auscultation important? Lancet 1982;2:1279

38. Grabowski JE, Talamini MA. Physiological effects of pneumoperitoneum. J Gastrointest Surg 2009;13:1009-16

39. Lestar M, Gunnarsson L, Lagerstrand L, Wiklund P, Odeberg-Wernerman S. Hemodynamic perturbations during robot-assisted laparoscopic radical prostatectomy in $45^{\circ}$ Trendelenburg position. Anesth Analg 2011;113:1069-75 